\title{
Taking the strain? Impact of glaucoma on patients' informal caregivers
}

\author{
Leanne McDonald $\mathbb{1}^{1,2} \cdot$ Paula Turnbull ${ }^{3} \cdot$ Lydia Chang $^{3} \cdot$ David P. Crabb $^{1}$
}

Received: 30 September 2019 / Revised: 5 November 2019 / Accepted: 6 November 2019 / Published online: 25 November 2019

(c) The Author(s), under exclusive licence to The Royal College of Ophthalmologists 2019

\begin{abstract}
Purpose To estimate informal caregiver (ICG) strain in people from a glaucoma clinic.

Methods Patients with glaucoma were consecutively identified from a single clinic in England for a cross-sectional postal survey. The sample was deliberately enriched with a number of patients designated as having advanced glaucoma (visual field [VF] mean deviation worse than $-12 \mathrm{~dB}$ in both eyes). Patients were asked to identify an ICG who recorded a Modified Caregiver Strain Index (MCSI), a validated 13 item instrument scored on a scale of 0-26. Previous research has indicated mean MCSI to be $>10$ in multiple sclerosis and Parkinson's disease. All participants gave a self-reported measure of general health (EQ5D).

Results Responses from 105 patients (43\% of those invited) were analysed; only 38 of the 105 named an ICG. Mean (95\% confidence interval [CI]) MCSI was $2.4(1.3,3.6)$ and only three ICGs recorded a MCSI $>7$. The percentage of patients with an ICG was much higher in patients with advanced VF loss $(82 \%$; 9/11) when compared with those with non-advanced VF loss $(31 \% ; 29 / 94 ; p=0.001)$. Mean (standard deviation) MCSI was considerably inflated in the advanced patients (5.6 [4.9] vs 1.5 [2.2] for non-advanced; $p=0.040$ ). Worsening VF and poorer self-reported general health (EQ5D) of the patient were associated with worsening MCSI.

Conclusion ICG strain, as measured by MCSI, for patients with non-advanced glaucoma is negligible, compared with other chronic disease. ICG strain increases moderately with worsening VFs but this could be partly explained by worse general health in our sample of patients.
\end{abstract}

\section{Introduction}

Chronic open angle glaucoma (COAG) can cause slow, irreversible damage to the visual field (VF). COAG, like many other chronic conditions affecting older adults, does not limit lifespan but can make life more challenging. For example, patients can report significant problems with activities of daily living, such as driving, reading and mobility as their VF worsens $[1,2]$. Such difficulties may

Leanne McDonald

leanne.mcdonald@uwl.ac.uk

1 Optometry and Visual Science, City, University of London, London, UK

2 Psychology, School of Human and Social Sciences, University of West London, London, UK

3 Department of Ophthalmology, North West Anglia NHS Foundation Trust, Hinchingbrooke Hospital, Huntingdon, UK lead to a reliance on a spouse, partner, close friend or family member for support [3,4]. A person caring for someone with a chronic or disabling condition, but not in a formal capacity, can be termed an informal caregiver (ICG).

Informal caregiving, much like the condition that the patient is experiencing, can be a complex issue incorporating physical, psychological, financial and emotional changes [5]. When these experiences are negative, it is termed caregiver strain [6]. For example, informal caregivers have been shown to experience exhaustion, problems with wellbeing and reduced levels of self-esteem [7]. ICG strain is most likely to affect women and those who do not have adequate social support [8,9]. Conversely, ICGs who are psychologically well adjusted, have good social support and implement proactive coping strategies are less likely to suffer from ICG strain [6]. ICGs often do not report their caregiver status to healthcare professionals and as such may not receive appropriate support [10].

ICG strain is well studied in conditions like cancer [11] and mental illness [12] where burden of care is often 
significant. More recently, ICG strain in long-term conditions has received attention. For example, ICG strain in Parkinson's disease (PD) and multiple sclerosis (MS) was found to be significant when measured quantitatively using a modified version of the Caregiver Strain Index (MCSI) [6]. The same may be true for long-term chronic eye conditions. For example, ICG strain has recently been described in people with age-related macular degeneration (AMD) especially as the condition leads to visual impairment [1317]. Moreover, specific aspects of ICG strain for AMD, like that associated with frequent treatment visits to clinic, have been flagged [14, 16]. There has also been an effort to assess ICG strain in paediatric glaucoma patients [18, 19]. However, there has been no attempt to quantify ICG strain in adult COAG and this is the main idea presented in this paper.

We estimate ICG strain in people in a glaucoma clinic in England. We do this with a cross-sectional study using a widely used and well validated standardised instrument (MCSI) [20]. We primarily aim to compare values from this index to values from other chronic conditions where ICG strain has been investigated using the same measure, specifically those described in Peters et al. [6]. We test a secondary hypothesis that measures of worsening VF in COAG are associated with worsening ICG strain as measured by MCSI.

\section{Materials and methods}

We designed a cross-sectional study involving patients recruited from the glaucoma clinic of Hinchingbrooke Hospital (part of North West Anglia NHS Foundation Trust). The study was approved by the NHS Research and Ethics committee of the East of Scotland (17/ES/0044 ref number: 216487) and adhered to the tenets of the Declaration of Helsinki. Patient participants were selected consecutively from an Electronic Medical Record (EMR) (Medisoft, Leeds, UK) by the study coordinator (PT) and the clinic's main glaucoma consultant (LC). To be eligible, patients ( $>40$ years) had to be currently treated for a diagnosis of COAG with VF loss in at least one eye. COAG suspects and patients with ocular hypertension were excluded. Participants were only included if they had no other ocular disease (except for uncomplicated cataract extraction) and a corrected binocular visual acuity (VA) of better than $\operatorname{LogMAR} 0.3(6 / 12)$ at their last clinic visit. Patients were selected consecutively from the date they last attended clinic, and this had to be within 6 months of the data extraction. Names and addresses were recorded along with age (years) and a measure of VF loss in both eyes (mean deviation; MD) from their last clinic visit as acquired using a Humphrey Field Analyser (Carl Zeiss Meditec,
Dublin, CA). The EMR also has a field for the number of significant non-ocular co-morbidities and this number was recorded too.

We aimed to select a total of 250 patients representing a population of people with COAG being treated in a clinic in England (see data analysis; sample size). We deliberately aimed to include 50 patients (some selected non-consecutively) designated as having advanced COAG defined as MD worse than $-12 \mathrm{~dB}$ in both eyes. This measure for advanced VF loss has been widely used before in, for example, health economic evaluations of COAG and coincides with a high-likelihood that the patient does not satisfy the VF component for legal fitness to drive [21, 22].

A questionnaire pack, including a participant information document, was posted to the address of selected participants. Due to the postal nature of the survey, participants were asked to complete a statement of implied consent. The patient information document asked participants to identify an ICG (if applicable) with the following question: 'Can you identify someone who is an informal caregiver for your glaucoma? This might be a spouse, a partner, a relative or friend who helps you with any aspect related to your glaucoma.'

The questionnaire pack included two sections printed on different coloured paper, one for the patient and one for their potential ICG. The patient section had demographic questions and a validated instrument (EQ5D) to measure self-reported general health. EQ5D is commonly used by NICE (The National Institute for Health and Care Excellence) for health economic evaluations for clinical interventions. We used EQ5D-5L in which items are scored from 1 (no problems) to 5 (severe problems) on the five domains of mobility, self-care, usual activities, pain/discomfort and anxiety/depression. An EQ5D index score was generated in a standard way with 1 representing full health (a score of one on all five items), and on the basis of a socalled UK tariff (applicable to our participants), a worst health state of -0.594 [23].

If an ICG was identified by the patient, then they completed a separate section of the questionnaire with its own consent statement; this included demographic questions, the EQ5D and MCSI questionnaires [20]. MCSI has been widely used with more than 200 citations in the literature. MCSI estimates levels of ICG strain in terms of financial, physical, psychological, social, and personal strain using 13 items, each of which is scored 'yes, regularly', 'yes, sometimes' or 'no'. Scores range from 0 ('no' on all items) to 26 ('yes, regularly' on all items).

The questionnaire pack was sent with two stampedaddressed envelopes to ensure that responses could be returned anonymously. A 'thank you' note/reminder was sent two weeks later to encourage responses. Data from the questionnaires was double entered. Median imputation was 
used for any missing values. Data was anonymised and stored in a secure location.

\section{Data analysis}

Our primary outcome was mean MCSI in the ICGs of the participating patients and a comparison with values reported from a study by Peters et al. for ICGs for people with MS and PD [6]; these values were 11 and 12, respectively. We aligned our study to the one by Peters et al. because it used MCSI on large numbers of ICGs for people with chronic conditions. From that study the between person standard deviation (SD) for MCSI was six units. Therefore, a samplesize calculation for a one-sample $t$-test aiming to demonstrate a difference of at least two units between mean MCSI in our data as compared with ones described in Peters et al. (power and alpha set at 0.80 and 0.05 , respectively) required at least 75 ICG responses. Assuming a response rate of $30 \%$ (Peters et al. had 37\%) meant we aimed to post 250 questionnaire packs.

Our secondary aims were to compare MCSI between ICGs of patients with and without advanced VF loss, and then to explore the association between MCSI and worsening COAG as measured by VF loss corrected for other measures such as, for example, sex, age and self-reported general health (EQ5D). Two-sample $t$-tests (assumed unequal variances) were used to compare means and Chi-square tests were used for categorical values. Associations were explored with Pearson correlation coefficients and a generalised linear model to correct for covariance. A value of 0.05 was used for statistical significance. Analysis was done in SPSS Statistics 23 (IBM Corp., Somers, NY) and in R (R Foundation for Statistical Computing, Vienna, Austria).

\section{Results}

We sent invitations to 243 patients, falling short of enriching our sample with our target of inviting 50 patients with advanced COAG $(n=39)$; finding eligible patients fulfilling the advanced VF criteria with preserved VA or not having other ocular pathology was problematic. Onehundred and sixteen (48\%) patients responded. Median (interquartile range) time period between a questionnaire pack being posted and returned was 14 (725) days.

Mean (SD) age of the patients who responded $(n=116)$ to the postal survey was $73(10)$ years. Mean (SD) better eye MD (BEMD) of the patients who responded was -3.7 (6.4) dB. Nine patients returned questionnaires declining to take part. Two other patients were not analysed: on checking data entry of the clinical record one was found not to satisfy the inclusion criteria for VA and the other had too many missing items to be analysed meaningfully. This left 105 patients for data analysis.
Only $38(36 \%)$ of the 105 patients analysed had an ICG. These patients represent just $16 \%$ of the total of $n=243$ contacted, a value lower than we anticipated in our samplesize calculations $(30 \%)$ perhaps reflecting that most people in glaucoma clinics do not consider their condition warrants an ICG. This in itself is an important finding in relation to the conditions like PD and MS investigated by Peters et al. [6] because in that study response rates were higher.

A participant stating that they had an ICG might be related to whether they are married or have a partner. For example, in the patients with an ICG, 87\% (33/38) selfreported they were married or in a committed relationship as opposed to being single, divorced, widowed or separated; in contrast this proportion was $60 \%(40 / 67)$ in the patients who did not have an ICG and the difference was statistically significant $(p=0.004)$. Percentage of male participants with and without an ICG was 47\% (18/38) and 55\% (37/67), respectively; these values were not significantly different $(p$ $=0.439$ )

Our primary outcome for the study was mean (SD) MCSI; this was 2.4 (3.4) in the 38 ICGs who completed the questionnaire (95\% CI: $1.3,3.6)$. This value was overwhelmingly statistically different $(p<0.001$, one-sample $t$ test) from the mean value of $\sim 11$ reported in ICGs for people with MS and PD in Peters et al[6]. Moreover, nearly one-half $(n=18 ; 47 \%)$ of our sample of ICG respondents returned a MCSI of zero (indicating no ICG strain, responding negatively to all 13 items). Furthermore, only three ICGs recorded a MCSI > 7, a value that some studies have described as meaningful caregiver strain. Taken together these results suggest ICG strain in COAG, as measured by MCSI, is negligible for most of the ICGs of COAG patients.

Mean (SD) best eye MD in patients with $(n=38)$ and without $(n=67)$ an ICG was $-6.9(9.1) \mathrm{dB}$ and $-2.1(4.0)$ $\mathrm{dB}$, respectively; these values are statistically different $(p=$ $0.004)$ hinting ICG strain increases with worsening VF loss. Moreover, percentage of patients with an ICG was much higher in patients with advanced VF loss $(82 \%$; 9/11) when compared with those with non-advanced VF loss (31\%; 29 / $94)$ and this difference was statistically significant $(p=$ 0.001). To further highlight this effect of ICG strain being inflated in advanced COAG, Table 1 gives the patient participant and ICG response stratified by our measure of COAG severity. For example, ICG mean (SD) MCSI was much worse when the patient had advanced VF loss (5.6 [4.9]). The three ICGs with MCSI $>7$ were for patients advanced VF loss too; this is noteworthy. There was no real evidence to suggest that the sex and age profile, or number of co-morbidities, of the two groups of patients were different. Yet patients with advanced VF loss, and their ICGs, had worse self-reported general health (EQ5D) compared with the others in the clinic and their respective ICGs. 
Table 1 Comparison between patients with and without advanced VF loss and their respective ICG responses

\begin{tabular}{lccc}
\hline & $\begin{array}{l}\text { Patients }(n=9) \text { with } \\
\text { advanced VF loss }\end{array}$ & $\begin{array}{l}\text { Patients }(n=29) \text { with } \\
\text { non-advanced loss }\end{array}$ & $p$ value \\
\hline Patient age (years) & $78(9)$ & $72(7)$ & 0.077 \\
Patient: female & $5(56 \%)$ & $15(52 \%)$ & 0.841 \\
Better eye mean deviation dB & $-21.5(6.1)$ & $-2.4(3.2)$ & $<0.001^{*}$ \\
Worse eye mean deviation dB & $-26.5(4.9)$ & $-6.8(5.3)$ & $<0.001^{*}$ \\
Patient: EQ5D score & $0.66(0.21)$ & $0.87(0.15)$ & $0.018^{*}$ \\
Patient: number of co-morbidities & $1.9(2.8)$ & $1.5(1.4)$ & 0.690 \\
Modified Care Strain & $5.6(4.9)$ & $1.5(2.2)$ & $0.040^{*}$ \\
Index (MCSI) & $5(56 \%)$ & $13(45 \%)$ & 0.573 \\
Informal caregiver (ICG): & & & $<0.001^{*}$ \\
number of females & $0.77(0.07)$ & & \\
Informal caregiver (ICG): & & & \\
EQ5D score & & & \\
\hline
\end{tabular}

Means with standard deviations ( $p$ value for two-sample test [unequal variances]) and numbers with percentages ( $p$ value for Chi-square test) are given for the measurements and categorical values, respectively. (An asterisk denotes statistical significance at $p<0.05$ )
Table 2 Pearson correlation coefficients for different measured variables against MCSI in 38 patients with ICGs

\begin{tabular}{llc}
\hline & $\begin{array}{l}\text { Correlation } \\
\text { coefficient }(\mathrm{r})\end{array}$ & $p$ value \\
\hline Patient age (years) & +0.11 & 0.499 \\
Better eye mean deviation dB & -0.46 & $0.003^{*}$ \\
Worse eye mean deviation dB & -0.62 & $<0.001^{*}$ \\
Patient: EQ5D score & -0.53 & $0.001^{*}$ \\
Patient: number of co-morbidities & +0.31 & 0.063 \\
Informal caregiver (ICG): & -0.26 & 0.113 \\
EQ5D score & & \\
\hline
\end{tabular}

An asterisk denotes statistical significance at $p<0.05$

Associations of measured variables with worsening MCSI in the 38 patients with ICGs are shown in Table 2. Worsening VF and poorer self-reported general health (EQ5D) of the patient were highly associated with worsening ICG MCSI. This analysis was exploratory because our study was not powered for this. Still, no other variables had a statistically significant association with MCSI. Given the influence of patient EQ5D we returned to our comparison of mean ICG MCSI between the patients with advanced $(n=9)$ and non-advanced VF loss $(n=29)$ using a general linear model (sometimes referred to as ANCOVA). After controlling for EQ5D as a covariate the difference in MCSI between the two groups still remained statistically significant ( $p=0.035$ vs $p=0.001$ [unadjusted with equal variances assumed]) but the effect diminished with a mean $(95 \% \mathrm{CI})$ difference in MCSI of $2.7(0.2,5.2)$ reduced from $4.1(1.8$, 6.4) (unadjusted). This analysis still suggests having advanced VF loss inflates ICG strain but in our data this is partly explained by the same patients having a co-varying worse self-reported general health. Of course, worse general health may or may not be related to having advanced VF loss, but this cannot be untangled with our data.

MCSI items (questions) with the 38 ICG's responses are given in Table 3. One third of ICGs have at least sometimes made changes in personal plans because of their caregiving. Other relatively more common strains surrounded work adjustments and less time for other family members. MCSI items referring to disturbed sleep, physical strain and a feeling of being 'overwhelmed' were completely rejected by all but a few ICGs.

Some other results from our sample of participants are worth noting. Nearly all patients $(98 \% ; 103 / 105)$ were Caucasian and 38\% (40/105) self-reported being educated to degree level or higher. In our sample of 38 ICGs there were roughly equal numbers of men $(n=18)$ and women $(n=20)$; mean (SD) MCSI was similar $(p=0.606)$ for men (2.1[3.6]) and women (2.7 [3.4]) too.

\section{Discussion}

We used a cross-sectional postal survey to illicit a measure of ICG strain for glaucoma patients in a single clinic in England. Patients were selected consecutively but the sample was enriched with a number of patients with advanced VF loss. Only $36 \%$ of patients who responded felt they had an ICG and in these, caregiver strain as measured by a standardised instrument (MCSI) was negligible. Although, in a subset of patients with advanced VF loss in both eyes, but preserved VA and no other ocular comorbidity, the ICGs response on MCSI was considerably inflated. 
Table 3 The 13 items from the Modified Care Strain Index questionnaire ranked by the frequency of responses by the informal caregivers (ICGs)

\begin{tabular}{llll}
\hline & Not at all & Sometimes & Regularly \\
\hline There have been changes in personal plans because of my caregiving & $\mathbf{6 6}$ & 31 & 3 \\
There have been work adjustments because of my caregiving & $\mathbf{7 6}$ & 16 & 8 \\
Caregiving is confining/restricting & $\mathbf{7 4}$ & 26 & 0 \\
There have been other demands on my time (e.g. other family members & $\mathbf{7 6}$ & 24 & 0 \\
need me) which I have been unable to deal with & & & 3 \\
It is upsetting to find the person I care for has changed so much from & $\mathbf{7 9}$ & 18 & 0 \\
his/her former self & $\mathbf{8 2}$ & 18 & 0 \\
There have been family adjustments because of my caregiving & $\mathbf{8 4}$ & 16 & 3 \\
Caregiving is inconvenient & $\mathbf{8 7}$ & 10 & 0 \\
There have been emotional adjustments because of my caregiving & $\mathbf{8 7}$ & 13 \\
My caregiving is a financial strain & $\mathbf{8 9}$ & 8 & 3 \\
Some behaviour is upsetting (the person I care for has upsetting & & & \\
behaviours) & $\mathbf{8 9}$ & 11 & 0 \\
My sleep is disturbed by my caregiving & $\mathbf{9 2}$ & 8 & 0 \\
Caregiving is a physical strain & $\mathbf{9 2}$ & 8 & 0 \\
I feel completely overwhelmed by my caregiving &
\end{tabular}

The top and bottom item in the table represent the item cited as the most common and least common strain experienced by the ICGs, respectively. The numbers are rounded whole percent of the $n=38$ ICGS

Bold values indicate the number of participants that answered 'not at all' to the MCSI item
Results from this study represent new knowledge about ICG strain in glaucoma patients. Our data might be useful for clinicians and practitioners who may not have considered ICG in COAG before. A raised awareness is useful because there is evidence that ICGs who are given adequate support do not experience as much strain [10]. Moreover, our data might be useful for targeting patients who need extra support and also health economic models for glaucoma care [24].

Comparing MCSI values between different conditions seems attractive but is fraught with issues because of the different sampling and methodology used in different studies. For example, MCSI (not the modified version) $>7$ has been reported in $36 \%$ of ICGs of people recovering from hip fracture surgery [25], 15\% of ICGs of people with adult cancer [26] and 24\% of ICGs of people with mild relapsingremitting MS [27]. In contrast we only had three ICGs with MCSI > 7; this could be reported as 3/105 (3\%) of people who were contacted/replied, or 3/38 (8\%) of ICGs analysed or $3 / 9(33 \%)$ of the people with advanced COAG; these different figures illustrate how sampling can affect results. We aligned our results to Peters et al. [6] but even their study had different methodology to ours. Still, for our primary outcome, mean MCSI for ICGs of patients in glaucoma clinics was considerably lower than values estimated by Peters et al. for MS and PD.

Greater ICG strain being related to worse VFs is another novel finding of our study; the association was true in the least and most affected eye. Mean MCSI was three times larger in our sample of patients with advanced VF loss compared with other patients in the clinic; this co-varied by the patients self-reported general health (EQ5D) but the effect remained after statistically correcting for this. This result is unsurprising because studies have indicated a rapid decline in vision related quality of life in COAG as both eyes progress to end stage VF loss [28, 29] and this likely reflects the greater help these people need. Of course, our findings add to the evidence that halting VF progression is a clinical imperative, not just for the patient but also for the wellbeing of the ICG of a patient. A longitudinal study would be needed to explore how ICG strain increases as COAG progresses in an individual and this could untangle the effect from worsening of general health.

ICG strain in another age-related eye condition, AMD, has been explored but making comparison with these studies is also tricky. For example, a study specifically assessed people on ranibizumab (injection) therapy for neovascular AMD and found it was associated with significant ICG strain [14]. Other studies have highlighted ICG strain in AMD but none sampled consecutively from people in clinics nor used MCSI, so it is difficult to make comparisons [13, 15]. A large multicentre cross-sectional study conducted in Portugal demonstrated visual impairment, defined as worse than $0.30 \log$ MAR $(6 / 12)$ in the better seeing eye, incurs ICG strain [30]. Our results from patients with advanced VF loss add to this knowledge because they had inflated ICG but, because of our study design, their VA was better than $6 / 12$.

There are good explanations for why ICG strain was insignificant in the majority of our sample of patients. Many 
of these patients are receiving treatment for a condition that is almost always asymptomatic until advanced in nature. In addition, patients had relatively preserved VA and no other ocular morbidity. In addition, although MCSI is widely used it is unlikely to capture specific ICG strain for people with COAG. For example, it was obvious that some MCSI items (Table 3), like care being physically draining, were rejected. Analogous to this issue is the debate about items within patient reported outcome measures (PROMs) that are not glaucoma specific and how they might, for example, be insensitive to glaucoma progression [31, 32]. Investigating the specific aspects of ICG strain in COAG, with a view to the development of a condition specific measure would be a useful area for future work.

We speculate there may be ICG strain in COAG around the different treatments (drops/surgery) and this could be the subject of future work. Other idiosyncratic ICG strains for COAG might include the psychological burden of having a potentially blinding condition or loss of visual function that might restrict mobility or remove a driving licence. We know patients are very concerned about the latter [33] and this would likely impact on their ICG too. Qualitative analysis of interviews with patients and their ICGs could pinpoint these strains; this is a subject of further work by our lab. In turn, this research could lead to development of a simple COAG specific instrument that could be administered in a clinic to detect if there was a 'silent' developing ICG strain. Others have discussed the importance of identifying a precipice when patients lose self-medicating capability, and this might be identifiable with an appropriate instrument for the ICG [34].

Other results from our study are worth discussing. Any postal survey will suffer from non-response. Our main findings are likely unaffected by this but ICG strain could be different if everyone in the clinic was captured given the association of MCSI with disease severity. The high number of patients who declared not to have an ICG is interesting too. This might suggest that patients do not consider their COAG warrants an ICG. Yet we also found a strong link between having an ICG and being married or having a partner. In turn this highlights the importance of identifying patients who may be socially isolated or living on their own. Moreover, in our data we did not observe differences in the sex profile of the ICGs, with men and women reporting the same level of ICG strain. This contradicts studies where ICG strain has been thought to be something that affects women more than men $[8,9]$.

Our study had several strengths. We took advantage of a widely used, standardised instrument. In addition, our sampling was performed consecutively, and we measured other variables allowing for an analysis that corrected for covariates. At the same time our study has several limitations. We only sampled people from one centre; the patients were nearly all Caucasian and education levels were relatively high. Some studies have indicated that there may be cultural and ethnic differences in the experience of ICG strain [35]. VF records were extracted from an EMR and, although unlikely, may have changed in the maximum six-month period before a participant responded. Moreover, our study was only cross-sectional, relied on self-report and could only examine associations. Furthermore, a larger sample and conducting the study across more centres would have improved the generalisability of our results.

In conclusion, our study is novel in assessing ICG strain in patients from a glaucoma clinic. We conclude that ICG strain in the great majority of these patients is largely negligible but, importantly, it worsens as disease severity worsen. Patients with advanced VF loss in both eyes have considerably inflated ICG strain although some of this might be explained by worsening general health in these people too. Further work should be done to improve our understanding of the specific nuances of ICG in relation to $\mathrm{COAG}$.

\section{Summary}

\section{What was known before}

- Chronic open angle glaucoma (COAG) can cause difficulties with activities of daily living such as driving and mobility.

- Other chronic eye diseases, for example, age-related macular degeneration lead to an increase in reliance on family support networks; this is termed informal caregiving.

\section{What this study adds}

- The first to estimate caregiver strain in a sample of COAG caregivers using a validated instrument.

- ICG strain is negligible in most patients consecutively sampled from a glaucoma clinic in England.

- ICG strain becomes inflated in patients with advanced visual field (VF) loss in COAG.

- ICG strain increases moderately with worsening VF's but in our sample some of this could be explained by worse general health.

Funding This work was supported by an unrestricted investigator initiated grant from Santen UK and Santen International but they had no role in the design of the study, collection and analysis of data or decision to publish. 


\section{Compliance with ethical standards}

Conflict of interest The authors declare that they have no conflict of interest.

Publisher's note Springer Nature remains neutral with regard to jurisdictional claims in published maps and institutional affiliations.

\section{References}

1. Crabb DP. A view on glaucoma-are we seeing it clearly? Eye (Lond). 2016;30:304-13.

2. Glen FC, Crabb DP. Living with glaucoma: a qualitative study of functional implications and patients' coping behaviours. BMC Ophthalmol. 2015;15:128.

3. Shtein RM, Newman-Casey PA, Herndon L, Coleman AL, Lee PP. Assessing the role of the family/support system perspective in patients with glaucoma. J Glaucoma. 2016;25:e676-e680.

4. Keeffe JE, Chou SL, Lamoureux EL. The cost of care for people with impaired vision in Australia. Arch Ophthalmol. 2009;127: 1377-81.

5. Burleson Sullivan A, Miller D. Who is taking care of the caregiver? J Patient Exp. 2015;2:7-12.

6. Peters M, Jenkinson C, Doll H, Playford D, Fitzpatrick R. Carer quality of life and experiences of health services: a cross-sectional survey across three neurological conditions. Health Qual Life Outcomes. 2013;11:103.

7. van den Heuvel E, de Witte L, Schure L, Sanderman R, Meyboom-de Jong B. Risk-factors for burn-out in caregivers for stroke patients, and possibilities for intevention. Clin Rehabil. 2001;40:669-667.

8. Yee J, Schulz R. Gender differences in psychiatric morbidity among family caregivers: a review and analysis. Gerontologist. 2000;36:147-64.

9. McCullagh E, Brigstocke G, Donaldson N, Kalra L. Determinants of caregiving burden and quality of life in caregivers of stroke patients. Stroke. 2005;36:2181-6.

10. Royal College of General Practitioners and Royal College of Nursing. Matters of life and death: helping people to live well until they die. London: Royal College of General Practitioners and Royal College of Nursing; 2012. http://www.rcgp.org.uk/clinica 1-and-research/toolkits/ /media/Files/CIRC/Matters\%20of\% 20Life\%20and\%20Death\%20FINAL.ashx. Accessed Nov 2019.

11. Northouse LL, Katapodi MC, Schafenecker AM, Weiss D. The impact of caregiving on the psychological well-being of family caregivers and cancer patients. Semin Oncol Nurs. 2012;28: 236-45.

12. Chang S, Zhang Y, Jeyagurunathan A, Lau YW, Sagayadevan V, Chong SA, et al. Providing care to relatives with mental illness: reactions and distress among primary informal caregivers. BMC Psychiatry. 2016;16:80.

13. Schmier JK, Halpern MT, Covert D, Delgado J, Sharma S. Impact of visual impairment on use of caregiving by individuals with agerelated macular degeneraton. Retina. 2006;26:1056-62.

14. Gohil R, Crosby-Nwaobi R, Forbes A, Burton B, Hykin P, Sivaprasad S. Caregiver burden in patients recieving ranibizumab therapy for neovascular macular degeneration. PLOS ONE. 2015;10:e 0129361.

15. Vukicevic M, Heraghty J, Cummins R, Gopinath B, Mitchell P. Caregiver perceptions about the impact of caring for patients with wet age-related macular degeneration. Eye (Lond). 2016;30:413-21.

16. Hanemoto T, Hikichi Y, Kikuci N, Kozawa T. The impact of different anti-vascular endothelial growth factor treatment regimens on reducing burden for caregivers and patients with wet age-related macular degeneration in a single-center real-world Japanese setting. PLOS ONE. 2017;12:e0189035.

17. Gopinath B, Craig A, Kifley A, Liew G, Bloffwitch J, van Vu J, et al. Implementing a multi-modal support service model for the family caregivers of persons with age-related macular degeneration: a study protocol for a randomised controlled trial. BMJ Open. 2017;7:e018204.

18. Gothwal K, Bharani S, Mandal SK. Glaucoma quality of life of caregivers of children with congenital glaucoma: development and validation of a novel questionnaire (CarCGQoL). Invest Ophthalmol Vis Sci. 2015;56:770-7.

19. Kantipuly A, Pillai MR, Shroff S, Khatiwala R, Raman GV, Krishnadas SR, et al. Caregiver burden in primary congenital glaucoma. Am J Ophthalmol. 2019. https://doi.org/10.1016/j.ajo. 2019.05.003

20. Thornton M, Travis SS. Analysis of the reliability of the modified caregiver strain index. J Gerontol B Psychol Sci Soc Sci. 2003;58: S127-S13.

21. Boodhna T, Crabb DP. More frequent, more costly? Health economic modelling aspects of monitoring glaucoma patients in England. BMC Health Serv Res. 2016;16:611.

22. Saunders LJ, Russell RA, Crabb DP. Practical landmarks for visual field disability in glaucoma. Br J Ophthalmol. 2012;96: $1185-9$.

23. van Hout $B$, Janssen MF. Interim scoring for the EQ-5D-5L: mapping the EQ-5D-5L to EQ-5D-3L value sets. Value Health. 2012;15:708-15.

24. van den Burg B, Al M, van Exel J, Koopmanschap M, Brouwer W. Economic valuation of informal care: conjoint analysis applied in a heterogeneous population of informal caregivers. Value Health. 2008;11:1041-50.

25. Ariza-Vega P, Ortiz-Pina M, Kristensen MT, Castellote-Caballero Y, Jimenez-Moleon JJ. High perceived caregiver burden for relatives of patients following hip fracture surgery. Disabil Rehabil. 2019;41:311-8.

26. Hsu T, Loscalzo M, Ramani R, Forman S, Popplewell L, Clark K, et al. Are disagreements in caregiver and patient assessment of patient health associated with increased caregiver burden in caregivers of older adults with cancer? Oncologist. 2017;22:1383-91.

27. van der Hiele K, van Gorp DAM, Heerings MAP, Jongen P, van der Klink JJL, Frequin STFM, et al. Caregiver strain among life partners of persons with mild disability due to relapsing-remitting multiple sclerosis. Mult Scler Rel Dis. 2019;31:5-11.

28. Peters D, Heijl A, Brenner L, Bengtsson B. Visual impairment and vision related quality of life in the early manigest glaucoma trial after 20 years of follow up. Acta Ophthalmol. 2015;93:745-52.

29. Jones L, Bryan SR, Crabb DP. Gradually then suddenly? Decline in vision-related quality of life as glaucoma worsens. J Opthalmol. 2017;2017:7 https://doi.org/10.1155/2017/1621640

30. Marques AP, Macedo AF, Hernandez-Moreno L, Ramos PL, Butt $\mathrm{T}$, Rubin $\mathrm{G}$, et al. The use of informal care by people with vision impairment. PLOS ONE. 2018;13:e0198631.

31. Skalicky SE, Lamoureux EL, Crabb DP, Ramulu PY. Patientreported outcomes, functional assessment, and utility values in glaucoma. J Glaucoma. 2019;28:89-96.

32. Jones L, Garway-Heath DF, Azuara-Blanco A, Crabb DP, UKGTS Investigators. Are patient self-reported outcome measures sensitive enough to be used as end points in clinical trials? Evidence from the United Kingdom Glaucoma Treatment Study. Ophthalmology. 2019;126:682-9.

33. Bhargava JS, Bhan-Bhargava A, Foss AJ, King AJ. Views of glaucoma patients on provision of follow-up care; an assessment of patient preferences by conjoint analysis. $\mathrm{Br} \mathrm{J}$ Ophthalmol. 2008;92:1601-5. 
34. Read S, Waterman H, Morgan JE, Harper RA, Spencer AF, Stanford P. Glaucoma, dementia, and the "precipice of care": transitions between states of medication adherence. Patient Prefer Adherence. 2018;12:1315-25.
35. Haley WE, Roth DL, Howard G, Safford MM. Caregiving strain and estimated risk for stroke and coronary heart disease among spouse caregivers. Differential effects by race and sex. Stroke. 2010;41:331-6. 